\title{
ASYMPTOTIC EXPANSIONS OF COULOMB WAVE FUNCTIONS*
}

\author{
BY \\ MILTON ABRAMOWITZ \\ Computation Laboratory, New York
}

Introduction. Coulomb wave functions are used in nuclear physics in problems involving collisions of charged particles. The general picture in nuclear phenomena was clarified considerably by the analysis of the process of alpha particle emission by naturally radioactive elements which was initiated by the work of Gamow and of Condon and Gurney. In the discussions of this problem the large decrease of the Coulomb wave functions which takes place between the nuclear boundary and the high distance edge of the Coulomb barrier is of primary importance. This large decrease is very sensitive to both the height and width of the Coulomb barrier. The main feature of Coulomb wave functions which was important in the early work just mentioned is the critical dependence of the wave function on barrier properties. It was possible to be sure of the qualitative correctness of the explanation and its adequacy in accounting for the Geiger-Nuttall relation without having exact values of Coulomb wave functions. Their approximate representations by the Jeffries-Wentzel-Kramers-Brillouin method showed that alpha particles escape as though they were leaking through the Coulomb barrier in accordance with the Schroedinger equation. In later work it became unsatisfactory to rely on these approximations because the interest in nuclear physics grew and included a new range of experimental facts concerned with light nuclei and new reactions for heavy nuclei and also because the effect of changing the values of the angular momentum assumed for the escape of alpha particles in the uranium, thorium, and actinium families became of importance. For reactions involving light particles it is desirable to have reasonably accurate values of Coulomb wave functions. This is because the decrease of the wave function on passing through the barrier is not an especially dominating effect and has to be considered pretty much on a par with other factors, such as formation of semi-stable states by the bombarded and bombarding particles or the energy dependence of escape probabilities of fragments, which has sometimes no marked relation to Coulomb repulsion. It has also proved possible to draw some reasonably accurate conclusions concerning the magnitude of nuclear forces and the range of distances within which they are effective by the analysis of experiments on the scattering of protons by protons. In this connection, experiments on the scattering of protons by deuterons can be used to give additional information. In the analysis of proton-proton scattering experiments it is necessary to use exact rather than approximate values of Coulomb wave functions. The experiments are being extended in many laboratories to include a wider range of energies, and the tabulations which have been made in the past no longer suffice for the analysis. It is very valuable therefore to have more formulas and expansions of a suitable character for the computation of Coulomb wave functions for new ranges of parameters. Although it would be doubtless possible to obtain all the necessary values by purely computational means, it is also very helpful to have analytic representations which enable a physicist to think about what one might expect without the examination of voluminous tabulations of numbers.

${ }^{*}$ Received May 27, 1948. The introductory material has been furnished by Professor Gregory Breit of Yale University. 
1. Statement of Problem. The Computation Laboratory of the National Applied Mathematics Laboratories, National Bureau of Standards is now engaged in the tabulation of the regular solution of the differential equation

$$
y^{\prime \prime}+\left\{1-2 \eta \rho^{-1}-L(L+1) \rho^{-2}\right\} y=0
$$

for $L=0$ and for $\rho$ and $\eta$ in the range from 0 to 10 . For physical applications, a solution is desired such that $y=F_{L}(\rho, \eta) \rightarrow \sin \left[\rho-\eta \ln 2 \rho+L \pi / 2+\sigma_{L}\right]$ where $\sigma_{L}=\arg$ $\Gamma(L+1+i \eta)$ as $\rho \rightarrow \infty$. If we define

$$
F_{0}(\rho, \eta)=C_{0} \rho \phi_{0}(\rho, \eta)=C_{0} \rho \sum_{n=1}^{\infty} a_{n}(\eta) \rho^{n-1},
$$

where $C_{0}$ is a normalization factor independent of $\rho$, it may be shown that the coefficients $a_{n}$ satisfy the following recurrence relations

$$
a_{1}=1, \quad a_{2}=\eta, \quad n(n-1) a_{n}=2 \eta a_{n-1}-a_{n-2} \quad(n=3,4, \cdots) .
$$

The power series (1.2) is satisfactory for computational purposes when $\rho$ and $\eta$ are small. However, for large values of $\rho$ and $\eta$ the convergence is slow and a large number of terms are needed. It is the purpose of this article to obtain a number of expansions which may be used for large values of $\rho$ and $\eta$.

2. Asymptotic expansion valid for $\rho \gg \eta$. When $L=0$, equation (1.1) becomes

$$
y^{\prime \prime}+\left(1-2 \eta \rho^{-1}\right) y=0 .
$$

This equation has been studied in considerable detail by T. Sexl ${ }^{1}$ and M. Frenkel. ${ }^{2}$ The results obtained by Sexl will serve as a starting point for our discussion. Particular solutions of (2.1) may be expressed as contour integrals, namely

$$
\begin{aligned}
& y_{1}=\frac{1}{2 \pi i} \int_{C_{1}} e^{\rho z}(z-i)^{i \eta-1}(z+i)^{-i \eta-1} d z, \\
& y_{2}=\frac{1}{2 \pi i} \int_{C_{2}} e^{\rho z}(z-i)^{i \eta-1}(z+i)^{-i \eta-1} d z,
\end{aligned}
$$

where the paths of integration are shown in the accompanying diagram. It can be shown that

$$
\rho \phi_{0}(\rho, \eta)=e^{\pi \eta} y_{1}+y_{2} .
$$

In order to obtain an expansion in reciprocal powers of $\rho$ Sexl employs the MellinBarnes integral representation of a solution of (2.1). We shall obtain this expansion directly from (2.2) and then show how $C_{0}$ must be chosen so that the solution $F_{0}(\rho, \eta)$ should have the desired asymptotic behavior as $\rho \rightarrow \infty$.

If we set $(z+i) \rho=v$ in the integral for $y_{1}$ we obtain

$$
y_{1}=\left\{\frac{e^{-i \rho}(2 i \rho)^{i \eta}}{(-2 i)}\right\} \frac{1}{2 \pi i} \int_{L} e^{v}\left(1-\frac{v}{2 i \rho}\right)^{i \eta-1} v^{-i \eta-1} d v
$$

${ }^{1}$ T. Sexl, Zur Theorie der bei der wellenmechanischen Behandlung des radioaktiven Zerfalls auftretenden Differentialgleichung, Zeit. für Phys. 56, (1929).

${ }^{2} \mathrm{M}$. Frenkel, Die asymptotischer Lösungen der in der Theorie der radioaktiven $\alpha$-Emission auftretenden Differentialgleichung, Zeit für Phys. 95, (May 1935). 
the path of integration being a circuit from $-\infty$ around $v=0$ and then back to $-\infty$. Making use of the relation ${ }^{3}$

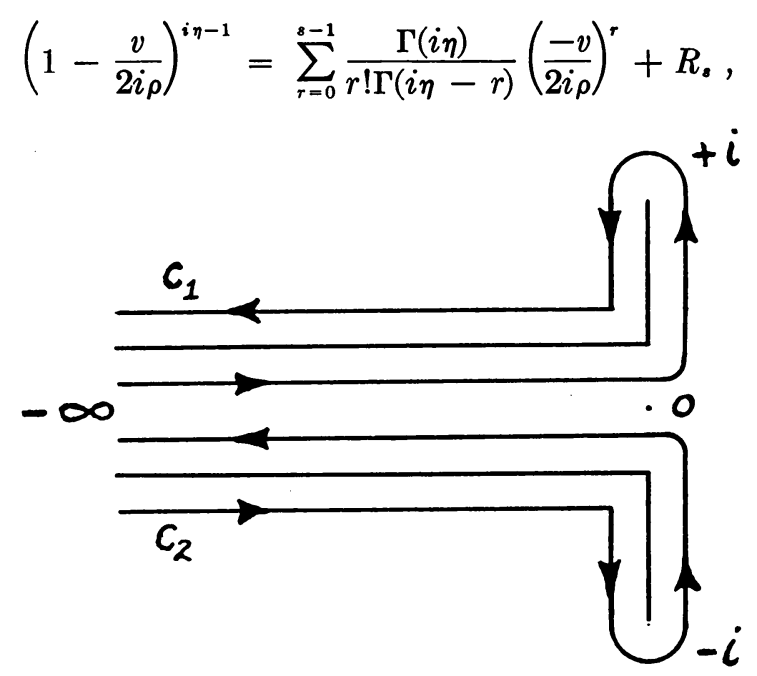

FIG. 1.

where

$$
R_{s}=\frac{\Gamma(i \eta)}{s ! \Gamma(i \eta-s)}\left(\frac{-v}{2 i \rho}\right)^{s} \int_{0}^{1} s(1-t)^{s-1}\left(1-\frac{v t}{2 i \rho}\right)^{i \eta-s-1} d t
$$

(2.4) becomes

$$
y_{1}=\left\{\frac{e^{-i \rho}(2 i \rho)^{i \eta}}{(-2 i \rho)^{s} s ! \Gamma(i \eta-s)}\right\}\left[\frac{1}{2 \pi i} \int_{L} \sum_{r=0}^{s-1} \frac{(-1)^{r} \Gamma(i \eta) e^{v} v^{r-i \eta-1}}{r ! \Gamma(i \eta-r)(2 i \rho)^{r}} d v+\bar{R} .\right]
$$

where

$$
\bar{R}_{s}=\frac{\Gamma(i \eta)}{(-2 i \rho)^{s} s ! \Gamma(i \eta-s)} \frac{1}{2 \pi i} \int_{L} e^{v} v^{-i \eta-1+s} d v \int_{0}^{1} s(1-t)^{s-1}\left(1-\frac{v t}{2 i \rho}\right)^{i \eta-s-1} d t .
$$

If in the above relation we invert the order of integration and summation and employ the relation ${ }^{4}$

$$
\frac{1}{2 \pi i} \int_{L} e^{v} v^{-n} d v=1 / \Gamma(n)
$$

we get finally

$$
y_{1}=\left\{\frac{e^{-i \rho}(2 i \rho)^{i \eta}}{(-2 i)}\right\}\left[\sum_{r=0}^{s-1} \frac{(-1)^{r} \Gamma(i \eta)}{(2 i \rho)^{r} r ! \Gamma(i \eta-r) \Gamma(i \eta+1-r)}+\bar{R}_{s}\right]
$$

The series (2.6) considered as an infinite series is divergent. However, if we truncate the series with the $s$-th term, it may be shown that $\left|\bar{R}_{s}\right|$ is of the order of $\left|\rho^{-s}\right|$ and

${ }^{3}$ T. M. MacRobert, Functions of a complex variable, Macmillan and Co., London, 1947, p. 271.

${ }^{4} \mathrm{R}$. Courant and D. Hilbert, Methoden der mathematischen Physik, Interscience Publishers, New York, 1943, vol. 1, p. 417. 
may be made as small as we please by taking $\rho$ sufficiently large. The proof is entirely similar to that for the Hankel function expansion ${ }^{5}$

The series (2.6) may be rewritten in the form

$$
y_{1}=\frac{e^{-i \rho}(2 i \rho)^{i \eta}}{(-2 i) \Gamma(1+i \eta)} U(\rho, \eta)
$$

where

$$
U(\rho, \eta)=1-\frac{i \eta(i \eta-1)}{2 i \rho}+\frac{i \eta(i \eta-1)^{2}(i \eta-2)}{2 !(2 i \rho)^{2}}-\cdots .
$$

It is clear that to obtain the equivalent result for $y_{2}$ we need only change $+i$ to $-i$ in (2.7). We then find

$$
y_{2}=\frac{e^{i \rho}(-2 i \rho)^{-i \eta}}{(2 i) \Gamma(1-i \eta)} \bar{U}(\rho, \eta)
$$

where $\bar{U}(\rho, \eta)$ is the complex conjugate of $U(\rho, \eta)$.

Now, let $\Gamma(1+i \eta)=|\Gamma(1+i \eta)| e^{i \sigma_{0}}$ so that (2.7) may be written

$$
y_{1}=\frac{U(\rho, \eta) e^{-\pi \eta / 2}}{(-2 i)|\Gamma(1+i \eta)|} \exp \left\{-i\left(\rho-\eta \ln 2 \rho+\sigma_{0}\right)\right\}
$$

To transform (2.9) in a similar manner we make use of the relation ${ }^{6}$

$$
\sigma_{0}=\arg \Gamma(1+i \eta)=-\gamma \eta+\sum_{n=1}^{\infty}\left(\frac{\eta}{n}-\arctan \frac{\eta}{n}\right)=-\arg \Gamma(1-i \eta),
$$

where $\gamma=.577215665 \cdots$ is Euler's constant, and the relation ${ }^{7}$

$$
|\Gamma(1+i \eta)|=|\Gamma(1-i \eta)|=\left(\frac{2 \pi \eta}{e^{\pi \eta}-e^{-\pi \eta}}\right)^{1 / 2}
$$

and obtain

$$
y_{2}=\frac{\bar{U}(\rho, \eta) e^{\pi / 2}}{(2 i)|\Gamma(1-i \eta)|} \exp \left\{i\left(\rho-\eta \ln 2 \rho+\sigma_{0}\right)\right\} .
$$

Making use of (2.3), (2.10), and (2.13) we finally get

$$
\rho \phi_{0}(\rho, \eta)=e^{\pi \eta} y_{1}+y_{2}=\left\{\frac{\bar{U} e^{i \varphi}-U e^{-i \varphi}}{2 i}\right\}
$$

where $\varphi=\rho-\eta \ln 2 \rho+\sigma_{0}$. Thus, if $U=u+i v$, we get

$$
F_{0}(\rho, \eta)=C_{0} \rho \phi_{0}(\rho, \eta)=u \sin \varphi-v \cos \varphi
$$

where $C_{0}=e^{-\pi \eta / 2}|\Gamma(1+i \eta)|$ and where

$$
u=1+\frac{\eta}{2 \rho}+\frac{5 \eta^{2}-\eta^{4}}{2 !(2 \rho)^{2}}+\cdots ; \quad v=\frac{-\eta^{2}}{2 \rho}+\frac{4 \eta^{3}-2 \eta}{2 !(2 \rho)^{2}}-\cdots .
$$

${ }^{5}$ Ibid, p. 453 .

${ }^{6} \mathrm{E}$. Jahnke and F. Emde, Tables of functions, Dover Publications, New York, 1945, p. 10.

${ }^{7}$ T. J. I'A. Bromwich, An introduction to the theory of infinite series, Macmillan and Co., London, 1926, p. 474. 
It is interesting to note that the expression for $F_{0}(\rho, \eta)$ can be obtained from (2.1) by elementary methods. Since (2.1) reduces to $y^{\prime \prime}+y=0$ as $\rho \rightarrow \infty$, this suggests the substitution

$$
y=e^{ \pm i \rho} \rho^{\mp k} u(\rho, \eta) .
$$

The differential equation for the function $u$ is

$$
u^{\prime \prime}+\left( \pm 2 i \mp 2 k \rho^{-1}\right) u^{\prime}+\left\{(\mp 2 i k-2 \eta) \rho^{-1}+k(k \pm 1) \rho^{-2}\right\} u=0 .
$$

If we assume the expansion

$$
u=1+\frac{a_{1}}{\rho}+\frac{a_{2}}{\rho^{2}}+\cdots
$$

and substitute in $(2.17)$, we obtain

$$
\begin{aligned}
(\mp 2 i k-2 \eta) \rho^{-1}+\left\{a_{1}[\mp 2 i k-2 \eta \mp 2 i]+k(k \pm 1)\right\} \rho^{-2} \\
\\
+\left\{a_{2}[\mp 2 i k-2 \eta \mp 2 i]+a_{1}[k(k \pm 1) \pm 2 k]\right\} \rho^{-3}+\cdots=0 .
\end{aligned}
$$

Thus, if we set $k= \pm i \eta$, the coefficients $a_{1}, a_{2}, a_{3}, \cdots$ can be determined successively. The function $F_{0}(\rho, \eta)$ is then obtained by taking the proper linear combination of the solutions thus found.

The relation (2.15) provides a convenient method of calculating the zeros of the function $\rho \phi_{0}(\rho, \eta)$. Setting (2.15) equal to zero, we get

$$
\tan \varphi=v(\rho, \eta) / u(\rho, \eta)
$$

and therefore $\varphi=\rho-\eta \ln 2 \rho+\sigma_{0}=\operatorname{arc} \tan v / u+n \pi(n$, an integer). This relation can be formulated iteratively as a formula for calculating the zeros, namely

$$
\rho_{s}^{(n)}=\eta \ln 2 \rho_{s-1}^{(n)}-\sigma_{0}+n \pi+\arctan \left\{v\left(\rho_{s-1}^{(n)}, \eta\right) / u\left(\rho_{s-1}^{(n)}, \eta\right)\right\}
$$

Thus, if $\rho_{s-1}^{(n)}$ represents an approximation to $\rho^{(n)}$, a better approximation can be obtained from (2.20). A short table of these zeros is given below.

$\begin{array}{cccc}\eta & n=1 & n=2 & n=3 \\ 0.0 & 3.1416 & 6.2832 & 9.4248 \\ 0.5 & 4.4578 & 7.8928 & 11.214 \\ 1.0 & 5.8140 & 9.4746 & 12.942 \\ 1.5 & 7.1262 & 10.974 & 14.567 \\ 2.0 & 8.3957 & 12.403 & 16.110 \\ 2.5 & 9.6330 & 13.786 & 17.596 \\ 3.0 & 10.846 & 15.130 & 19.033\end{array}$

3. Further expansions for large values of $\rho$ or $\eta$. Examination of the expansions (2.7) and (2.15) shows that for suitable convergence it is necessary that $\rho$ shall be large with respect to $\eta$. In the following development we shall obtain other expansions valid for $\rho<2 \eta$ as well as expansions for $\rho>2 \eta$.

Sexl obtained approximations to the contour integrals $y_{1}$ and $y_{2}$ given in (2.2) by 
the method of saddle points. Taking the appropriate linear combination defined in (2.3) we obtain

$$
\begin{gathered}
\rho \phi_{0}(\rho, \eta) \cong \frac{1}{(8 \pi \eta)^{1 / 2}}\left(\frac{\rho}{2 \eta-\rho}\right)^{1 / 4} \exp \left\{[\rho(2 \eta-\rho)]^{1 / 2}\right. \\
\left.\quad+2 \eta \arcsin (\rho / 2 \eta)^{1 / 2}\right\} \quad \text { for } \quad \rho<2 \eta \\
\rho \phi_{0}(\rho, \eta) \cong \frac{e^{\pi \eta}}{(2 \pi \eta)^{1 / 2}}\left(\frac{\rho}{2 \eta-\rho}\right)^{1 / 4} \sin [h(\rho, \eta)] \quad \text { for } \quad \rho>2 \eta
\end{gathered}
$$

where

$$
h(\rho, \eta)=\frac{\pi}{4}+2 \eta \ln \left[\left(\frac{\rho}{2 \eta}\right)^{1 / 2}-\left(\frac{\rho-2 \eta}{2 \eta}\right)^{1 / 2}+[\rho(\rho-2 \eta)]^{1 / 2}\right]
$$

The approximations (3.1) and (3.2) can be obtained directly from the differential equation (2.1) except for the normalizing constants. The method to be described is such that improved approximations can be obtained by elementary methods.

4. Derivation of (3.1). To obtain (3.1) we first put $\rho=2 \eta t$ in (2.1). The resulting equation is

$$
y^{\prime \prime}+4 \eta^{2}\left(1-t^{-1}\right) y=0 .
$$

If we now let $y=\exp \varphi$ the differential equation for the function $\varphi$ is

$$
\varphi^{\prime 2}+\varphi^{\prime \prime}+4 \eta^{2}\left(1-t^{-1}\right)=0 .
$$

This equation can be solved if we take for $\varphi(\rho, \eta)$ an expansion of the form

$$
\varphi(t, \eta)=(2 \eta) g_{0}(t)+g_{1}(t)+\frac{1}{(2 \eta)} g_{2}(t)+\frac{1}{(2 \eta)^{2}} g_{3}(t)+\cdots .
$$

Substituting (4.3) into (4.2) and equating coefficients of the successive powers of $(2 \eta)$ to zero, we obtain the following systems of equations

$$
\begin{aligned}
& g_{0}^{\prime 2}+\left(1-t^{-1}\right)=0, \\
& g_{0}^{\prime \prime}+2 g_{0}^{\prime} g_{1}^{\prime}=0 \\
& g_{1}^{\prime \prime}+g_{1}^{\prime 2}+2 g_{0}^{\prime} g_{2}^{\prime}=0, \\
& g_{2}^{\prime \prime}+2 g_{1}^{\prime} g_{2}^{\prime}+2 g_{0}^{\prime} g_{3}^{\prime}=0
\end{aligned}
$$

Solving these systems successively for $g_{0}, g_{1}, g_{2}$, we find

$$
\begin{aligned}
& g_{0}=\frac{1}{2 \eta}[\rho(2 \eta-\rho)]^{1 / 2}+\arcsin (\rho / 2 \eta)^{1 / 2} \\
& g_{1}=\frac{1}{4} \ln \left(\frac{\rho}{2 \eta-\rho}\right) \\
& g_{2}=-\left(\frac{\rho}{2 \eta-\rho}\right)^{1 / 2}\left\{\frac{2 \rho^{2}-6 \eta \rho+9 \eta^{2}}{12 \eta(2 \eta-\rho)}\right\} .
\end{aligned}
$$

Further approximations can be obtained by solving (4.4) for $g_{3}, g_{4}, \ldots$ 
Comparison of (3.1) with $\exp \varphi$ where $\varphi$ is defined by (4.3) in conjunction with (4.5) shows that if in (4.3) the terms beyond $g_{1}$ are neglected the approximation agrees with (3.1) except for an arbitrary normalizing constant. This suggests using the constant in (3.1) for the approximation just obtained. Thus for $\rho<2 \eta$

$$
\rho \phi_{0}(\rho, \eta)=\frac{1}{(8 \pi \eta)^{1 / 2}}\left(\frac{\rho}{2 \eta-\rho}\right)^{1 / 4} \exp \{\varphi(\rho, \eta)\}
$$

where

$$
\begin{aligned}
\varphi(\rho, \eta)=2 \eta \arcsin & \left(\frac{\rho}{2 \eta}\right)^{1 / 2}+[\rho(2 \eta-\rho)]^{1 / 2} \\
& -\frac{1}{2 \eta}\left(\frac{\rho}{2 \eta-\rho}\right)^{1 / 2}\left\{\frac{2 \rho^{2}-6 \eta \rho+9 \eta^{2}}{12 \eta(2 \eta-\rho)}\right\}+\cdots .
\end{aligned}
$$

5. A second approximation to $\rho \phi_{0}(\rho, \eta)$ for $\rho<2 \eta$. Let

$$
y=N \exp \{\varphi(\rho, \eta)\}
$$

where $N$ is a constant to be determined subsequently. Substituting (5.1) into (2.1) we obtain

$$
\varphi^{\prime 2}+\varphi^{\prime \prime}+1-2 \eta \rho^{-1}=0 .
$$

Assume for $\varphi(\rho, \eta)$ an expansion of the form

$$
\varphi(\rho, \eta)=(2 \eta)^{1 / 2} g_{0}(\rho)+g_{1}(\rho)+(2 \eta)^{-1 / 2} g_{2}(\rho)+\cdots .
$$

Substituting in (5.2) and equating powers of $(2 \eta)^{1 / 2}$ to zero, we get

$$
\begin{aligned}
& g_{0}^{\prime 2}-\rho^{-1}=0, \\
& 2 g_{0}^{\prime} g_{1}^{\prime}+g_{0}^{\prime \prime}=0,
\end{aligned}
$$

These equations may be solved successively, and we obtain

$$
\begin{array}{ll}
g_{0}=2 \rho^{1 / 2}, & g_{3}=\rho^{-1}\left(\frac{\rho^{2}}{4}-\frac{3}{64}\right), \\
g_{1}=\frac{1}{4} \ln \rho, & g_{4}=-\rho^{-3 / 2}\left(\frac{\rho^{4}}{20}+\frac{\rho^{2}}{32}+\frac{21}{1024}\right) . \\
g_{2}=-\rho^{-1 / 2}\left(\frac{\rho^{2}}{3}+\frac{3}{16}\right), &
\end{array}
$$

The expression for $\rho \phi_{0}(\rho, \eta)$ is thus determined except for the arbitrary normalizing factor. To determine this factor we note that if $\rho \ll 2 \eta$, the relation (3.1) becomes

$$
\rho \phi_{0}(\rho, \eta) \cong \frac{1}{(8 \pi \eta)^{1 / 2}}\left(\frac{\rho}{2 \eta}\right)^{1 / 4} \exp \left[2(2 \eta \rho)^{1 / 2}\right] .
$$


Furthermore, if we neglect the terms beyond $g_{1}(\rho)$ in (5.5) the resulting approximation for $\rho \phi_{0}(\rho, \eta)$ is the same as (5.6). This suggests choosing $N=(8 \pi \eta)^{-1 / 2}(2 \eta)^{-1 / 4}$ in (5.1). The expression for $\rho \phi_{0}(\rho, \eta)$ now becomes

$$
\rho \phi_{0}(\rho, \eta)=\frac{1}{(8 \pi \eta)^{1 / 2}}\left(\frac{\rho}{2 \eta}\right)^{1 / 4} \exp [\varphi(\rho, \eta)]
$$

where

$$
\begin{gathered}
\varphi(\rho, \eta)=2(2 \eta \rho)^{1 / 2} \\
-\frac{1}{(2 \eta \rho)^{1 / 2}}\left(\frac{\rho^{2}}{3}+\frac{3}{16}\right)+\frac{1}{2 \eta \rho}\left(\frac{\rho^{2}}{4}-\frac{3}{64}\right) \\
-\frac{1}{(2 \eta \rho)^{3 / 2}}\left(\frac{\rho^{4}}{20}+\frac{\rho^{2}}{32}+\frac{21}{1024}\right)+\cdots
\end{gathered}
$$

6. A third approximation to $\rho \phi_{0}(\rho, \eta)$ for $\rho<2 \eta$. The expression (5.7) suggests still another possible form of solution. We assume that

$$
\rho \phi_{0}(\rho, \eta)=\frac{1}{(8 \pi \eta)^{1 / 2}}\left(\frac{\rho}{2 \eta}\right)^{1 / 4} \exp \left[2(2 \eta \rho)^{1 / 2}\right] u(\rho, \eta)
$$

so that $u(\rho, \eta)$ must be a solution of the differential equation

$$
u^{\prime \prime}+\left(2(2 \eta / \rho)^{1 / 2}+\frac{1}{2 \rho}\right) u^{\prime}+\left(1-\frac{3}{16 \rho^{2}}\right) u=0 .
$$

If we now assume that $u$ may be expressed in the form

$$
u=u_{0}+\frac{u_{1}}{(2 \eta)^{1 / 2}}+\frac{u_{2}}{2 \eta}+\frac{u_{3}}{(2 \eta)^{3 / 2}}+\cdots,
$$

we get the following system of equations

$$
\frac{2}{\rho^{1 / 2}} u_{n+1}^{\prime}+u_{n}^{\prime \prime}+\frac{1}{\rho} u_{n}^{\prime}+\left(1-\frac{3}{16 \rho^{2}}\right) u_{n}=0, \quad n=1,2,3, \cdots
$$

with $u_{0}=1$ determined by the form of (6.1). Solving (6.3) successively we get

$$
\begin{aligned}
& u_{1}=-\rho^{-1 / 2}\left(\frac{\rho^{2}}{3}+\frac{3}{16}\right), \\
& u_{2}=\rho^{-1}\left(\frac{\rho^{4}}{18}+\frac{7 \rho^{2}}{16}-\frac{3}{512}\right), \\
& u_{3}=-\rho^{-3 / 2}\left(\frac{\rho^{6}}{162}+\frac{89}{480}+\frac{179 \rho^{2}}{512}+\frac{13}{8192}\right) .
\end{aligned}
$$

7. Approximation to $\rho \phi_{0}(\rho, \eta)$ for $\rho>2 \eta$. We now proceed to obtain an approximation to $\rho \phi_{0}(\rho, \eta)$, valid for $\rho>2 \eta$. If in $(2.1)$ we make the substitution $t=(\rho-2 \eta) / 2 \eta$ the resulting differential equation becomes

$$
y^{\prime \prime}+\frac{4 \eta^{2} t}{1+t} y=0
$$


To derive the solution from this equation, the expression (3.2) suggests that we assume an expansion of the form $y=\exp [H(t, \eta)]$ where

$$
H(t, \eta)=\frac{\pi}{4}+2 \eta g_{0}(t)+g_{1}(t)+\frac{1}{2 \eta} g_{2}(t)+\cdots .
$$

The solution of (7.1) thus reduces once more to the solution of a system of equations to be solved successively and we ultimately get

$$
\begin{aligned}
& g_{0}=i \ln \left\{(t+1)^{1 / 2}-t^{1 / 2}+[t(t+1)]^{1 / 2}\right\}, \\
& g_{1}=\frac{1}{4} \ln \left\{\frac{t+1}{t}\right\}, \\
& g_{2}=-i\left(\frac{1+t}{t}\right)^{3 / 2}\left\{\frac{8 t^{2}+4 t+5}{48(1+t)^{2}}\right\} .
\end{aligned}
$$

The desired approximation to $\rho \phi_{0}(\rho, \eta)$ corresponds to the imaginary part of exp $[H(t, \eta)]$. Thus if $t=(\rho-2 \eta) / 2 \eta$ using the constant in (3.2) we find

$$
\rho \phi_{0}(\rho, \eta)=\frac{e^{\pi \eta}}{(2 \pi \eta)^{1 / 2}}\left(\frac{t+1}{t}\right)^{1 / 4} \sin [H(t, \eta)]
$$

where

$$
\begin{gathered}
H(t, \eta)=\frac{\pi}{4}+2 \eta \ln \left\{(t+1)^{1 / 2}-t^{1 / 2}+[t(t+1)]^{1 / 2}\right\} \\
-\frac{1}{2 \eta}\left(\frac{1+t}{t}\right)^{3 / 2}\left\{\frac{8 t^{2}+4 t+5}{48(1+t)^{2}}\right\}+\cdots
\end{gathered}
$$

8. Development for $2 \eta<1$. We shall now obtain an expansion valid for $2 \eta<1$ and for moderate $\rho$. Assume that a solution of (2.1) can be expressed in the form

$$
y=y_{0}+(2 \eta) y_{1}+(2 \eta)^{2} y_{2}+\cdots .
$$

Since this function must be identical with $\rho \phi_{0}(\rho, \eta)$, and since $\rho \phi_{0}(\rho, \eta)=0$ and $d / d \rho\left\{\rho \phi_{0}(\rho, \eta)\right\}=1$ for $\rho=0$ we shall require that $y(0)=0, y^{\prime}(0)=1, y_{n}(0)=0$, $y_{n}^{\prime}(0)=0$ for $n \geq 1$. Substituting in (2.1) we then get

$$
\begin{aligned}
& y_{0}^{\prime \prime}+y_{0}=0, \\
& y_{n}^{\prime \prime}+y_{n}=\frac{1}{\rho} y_{n-1} \quad \text { for } \quad n=1,2,3, \cdots .
\end{aligned}
$$

Solving this system of equations, we get

$$
\begin{gathered}
y_{0}=\sin \rho=\rho-\frac{\rho^{3}}{3 !}+\frac{\rho^{5}}{5 !}-\cdots, \\
y_{1}=a_{2} \frac{\rho^{2}}{2 !}-a_{4} \frac{\rho^{4}}{4 !}+\cdots, \\
a_{2}=1, \quad a_{4}=a_{2}+1 / 3, \quad a_{6}=a_{4}+1 / 5, \cdots
\end{gathered}
$$




$$
\begin{gathered}
y_{2}=b_{3} \frac{\rho^{3}}{3 !}-b_{5} \frac{\rho^{5}}{5 !}+\cdots, \\
b_{3}=1 / 2 a_{2}, \quad b_{5}=b_{3}+1 / 4 a_{4}, \quad b_{7}=b_{5}+1 / 6 a_{6}, \cdots \\
y_{3}=c_{4} \frac{\rho^{4}}{4 !}-c_{6} \frac{\rho^{6}}{6 !}+\cdots, \\
c_{4}=1 / 3 b_{3}, \quad c_{6}=c_{4}+1 / 5 b_{5}, \quad c_{8}=c_{6}+1 / 7 b_{7}, \cdots .
\end{gathered}
$$

The law of formation of the successive $y_{n}$ is straightforward.

9. Approximation in terms of Bessel functions for large $\eta$. A method similar to that employed in (8.1) may be used to obtain an expansion valid for large values of $\eta$ and for moderate $\rho$. We first make the substitution $t=2 \eta \rho$ in (2.1) and obtain

$$
y^{\prime \prime}-\frac{1}{t} y=-\frac{1}{4 \eta^{2}} y \text {. }
$$

Assume an expansion of the form

$$
y=k\left\{y_{0}(t)-\frac{1}{4 \eta^{2}} y_{1}(t)+\frac{1}{\left(4 \eta^{2}\right)^{2}} y_{2}(t)-\cdots\right\},
$$

where $k$ is a constant to be chosen so that $y^{\prime}(0)=1$. Substituting (9.2) in (9.1) we then obtain

$$
\begin{aligned}
& y_{0}^{\prime \prime}-t^{-1} y_{0}=0 \\
& y_{n}^{\prime \prime}-t^{-1} y_{n}=-y_{n-1}, \quad(n=1,2, \cdots) .
\end{aligned}
$$

The solution of (9.3) which is regular at $t=0$ is

$$
y_{0}=t^{1 / 2} I_{1}\left(2 t^{1 / 2}\right)=\sum_{n=0}^{\infty} \frac{t^{n+1}}{n !(n+1) !} .
$$

The equations (9.4) may be solved successively, and we find for $y_{1}$ and $y_{2}$

$$
\begin{aligned}
& y_{1}=\sum_{n=3}^{\infty} \frac{n^{3}-3 n^{2}+2 n+12}{3} \frac{t^{n}}{(n-1) ! n !}=\frac{2 t^{3}}{2 ! 3 !}+\frac{8 t^{4}}{3 ! 4 !}+\frac{20 t^{5}}{4 ! 5 !}+\cdots, \\
& y_{2}=\frac{24 t^{5}}{4 ! 5 !}+\frac{184 t^{6}}{5 ! 6 !}+\frac{784 t^{7}}{6 ! 7 !}+\frac{2864 t^{8}}{7 ! 8 !}+\frac{6784 t^{9}}{8 ! 9 !}+\cdots .
\end{aligned}
$$

It is clear from (9.5) that in order that the expansion (9.2) shall have the same slope as $\rho \phi_{0}(\rho, \eta)$ at $\rho=0$ that we must have $k=1 / 2 \eta$.

It should be mentioned that several of the above methods may be employed to derive expansions of (1.1) for integral values of $L$. 